\title{
Information Fusion and Discounting Techniques for Decision Support in Aerospace
}

\author{
Fiona Browne ${ }^{1}$, Yan $\mathrm{Jin}^{1}$, \\ David Bell ${ }^{2}$, Weiru Liu ${ }^{2} \&$ Colm Higgins ${ }^{1}$ \\ ${ }^{1}$ School of Mechanical \& Aerospace Engineering \\ ${ }^{2}$ School of Electronics, Electrical Engineering \& Computer Science \\ Queen's University Belfast \\ Email:\{f.browne, y.jin, da.bell,w.liu, c.j.higgins\}@qub.ac.uk
}

\author{
Niall Rooney \& Hui Wang \\ Artificial Intelligence \& Applications Research Group \\ School of Computing \& Mathematics \\ University of Ulster \\ Email:\{nf.rooney, h.wang\}@ulster.ac.uk
}

\begin{abstract}
Decision makers are required to make critical decisions throughout all stages of a life-cycle in large-scale projects. These decisions are important as they impact upon the outcome and the success of projects. In this paper we present an evidential reasoning framework to aid decision-makers in the decision making process. This approach utilizes the Dezert-Smarandache Theory (DSm) to fuse heterogeneous evidence sources that suffer from levels of uncertainty, imprecision and conflicts to provide beliefs for decision options. To analyze the impact that source reliability and priority has upon the decision making process a reliability discounting technique along with a priority discounting technique are applied. Application of the evidential reasoning framework is illustrated using a Case Study based in the Aerospace domain.
\end{abstract}

\section{INTRODUCTION}

Decision making in large-scale projects are often sophisticated and complex processes whereby selections have an impact on diverse stages of the project life-cycle and ultimately the outcome of the project. The Aerospace domain provides an example of such a problem space. This is a highly competitive field with constant demands on aircraft production to improve in terms of safety, performance, speed, reliability and cost effectiveness [1]. Design decisions made throughout an aircraft life-cycle are critical as they directly effect the factors above. Decision making in Aerospace involves the evaluation of multiple decision options against criteria such as detailed requirement specifications and International Aviation Standards specified for example, by the Federal Aviation Administration. Evidence supporting/opposing these options can be extracted from diverse heterogeneous information sources. However, these evidence vary in terms of reliability, completeness, precision and may contain conflicting information. To address these limitations we propose an evidential reasoning framework to support decision analysis using information fusion techniques based on Belief Function Theory to manage uncertainty and conflict in evidence sources. This research is an element of a larger, complex and collaborative project DEEPFLOW which encompasses the areas of natural language processing, high performance computing, computational semantics, and reasoning with uncertainty. The project aims to develop a framework to identify, extract and reason with information contained within large complex interrelated documents which can be applied to many diverse problem domains.

Bayesian methods and Evidence Theories such as DempsterShafer Theory (DS) [2] are commonly used to handle uncertainty. As a generalized probability approach DS has some distinct features compared with Bayesian theory. DS can represent ignorance caused by lack of information and can aggregate beliefs when new evidence is accumulated [3]. However, it has been demonstrated that DS is limited in decision making when the level of conflict between sources of evidence becomes high [4]. Dezert-Smarandache Theory (DSm) which overcomes the limitations of the DS approach is therefore applied in this paper to fuse evidence sources with DS presented for comparative purposes. DSm can be considered as a generalization of the DS whereby the rule of combination takes into account both uncertain and paradoxical information [5].

Evidence sources involved in the fusion process may not always have equal reliability or priority. This paper regards reliability and priority as distinct factors. Reliability can be viewed as an objective property of an evidence source whereas priority is viewed as a subjective property expressed by the fusion system designer/expert [6]. Counter-intuitive results could be obtained if unequal sources are fused and these factors are not taken into consideration. To address and highlight the importance of these factors in the decision making process we apply two discounting techniques: reliability discounting using the classical Shafer's discounting approach and priority discounting based on the importance discounting technique described in [6].

The paper is organized as follows: in section 2 the basics of Evidence Theory and combination rules are introduced. In section 3 the reliability discounting technique and our proposed priority discounting technique are detailed. A Case Study in section 4 presents an applied scenario based in the Aerospace domain comparing both the DS and DSm approaches and the impact of discounting factors on decision analysis. Conclusions and future work are provided in section 5. 


\section{BASICS ON DS AND DSM THEORY}

The DS (evidential theory) is a generalization of traditional probability. This theory provides a mathematical formalism to model our belief and uncertainty on possible decision options for a given decision making process. The application of the Dempster-Shafer rule of combination of belief functions has been advantageous in the fusing of uncertain evidence supporting different hypotheses [5]. However, when conflict between sources becomes high the DS can generate errors in decision making. To address this problem we use the DSm which can be considered a generalization of DS. DSm overcomes limitations of DS by proposing new models for the frame of discernment and new rules of combination that take into account both paradoxical and uncertain information. In DSm the elements $\theta_{i}, i=1,2, \ldots, n$ of the frame of discernment $\Theta$ are exhaustive but not necessarily exclusive due to the intrinsic nature of its elements. In comparison, DS considers that the frame $\Theta$ consists of a set of finite exhaustive and exclusive elements requiring refinement to ensure $\Theta$ is exclusive. DS works with a power-set of $\Theta$ denoted $2^{\Theta}$ whereas DSm uses a hyper power-set $D^{\Theta}$ (Dedekind's lattice) described in detail in [7] created with $\cup$ and $\cap$ operators.

A generalized Basic Belief Assignment (bba) expressing belief assigned to the elements of $D^{\Theta}$ provided by an evidential source (defined in a similar manner as DS in [2]) is a mapping function $m: D^{\Theta} \rightarrow[0,1]$ representing the distribution of belief satisfying the conditions:

$$
m(\emptyset)=0 \text { and } \sum_{A \in D^{\ominus}} m(A)=1
$$

In evidence theory, a probability range is used to represent uncertainty. The lower bounds of this probability is called Belief(Bel) and the upper bounds Plausibility $(\boldsymbol{P l})$. The generalized $B l$ and the $P l$ for any proposition $A \in D^{\Theta}$ can be obtained by:

$$
\operatorname{Bel}(A)=\sum_{\substack{B \subseteq A \\ B \in D^{\Theta}}} m(B) \text { and } \operatorname{Pl}(A)=\sum_{\substack{B \cap A \neq 0 \\ B \in D^{\Theta}}} m(B)
$$

In this paper, the DS frame $\Theta$ is used i.e. all elements $\theta_{i}$ of $\Theta$ are assumed to be exhaustive and exclusive. Therefore $D^{\Theta}$ is equal to $2^{\Theta}$ and the generalized belief functions are reduced to the classical DS functions.

In DSm the Proportional Conflict Redistribution Rule no. 5 (PCR5) has been proposed as an alternative to the DempsterShafer rule of combination for combining highly conflicting sources of evidence. Below the Dempster-Shafer rule of combination and PCR5 are briefly detailed, a complete presentation of DSm including examples can be found in [7].

\section{A. Dempster-Shafer Rule of Combination}

In DS, the Dempster-Shafer rule of combination symbolized by the operator $\oplus$ is used to fuse two distinct sources of evidence $B_{1}$ and $B_{2}$ over the same frame $\Theta$. Let $B e l_{1}$ and $\mathrm{Bel}_{2}$ represent two belief functions over the same frame $\Theta$ and $m_{1}$ and $m_{2}$ their respective bba. The combined belief function $\mathrm{Bel}=\mathrm{Bel}_{1} \oplus \mathrm{Bel}_{2}$ is obtained by the combination of $m_{1}$ and $m_{2}$ as: $m(\emptyset)=0$ and $\forall C \neq \emptyset \subseteq \Theta$

$$
m(C) \equiv\left[m_{1} \oplus m_{2}\right](C)=\frac{\sum_{A \cap B=C} m_{1}(A) m_{2}(B)}{1-\sum_{A \cap B=\emptyset} m_{1}(A) m_{2}(B)}
$$

The Dempster-Shafer rule of combination is associative $\left(\left[m_{1} \oplus\right.\right.$ $\left.\left.m_{2}\right] \oplus m_{3}=m_{1} \oplus\left[m_{2} \oplus m_{3}\right]\right)$ and commutative $\left(m_{1} \oplus m_{2}=\right.$ $\left.m_{2} \oplus m_{1}\right)$.

\section{B. PCR5 Rule of Combination}

In DSm the PCR5 rule is generally used to combine bba's. PCR5 transfers the conflicting mass only to those elements that are involved in the conflict and proportionally to their individual masses. This preserves the specificity of the information in the fusion process [8]. For two independent bba's $m_{1}$ and $m_{2}$ the PCR5 rule defined by [7] is as follows: $m_{P C R 5}(\emptyset)=0$ and $\forall(X \neq \emptyset) \in 2^{\Theta}$

$$
\begin{gathered}
m_{P C R 5}(A)=\sum_{\substack{X_{1}, X_{2} \in 2^{\Theta} \\
X_{1} \cap X_{2}=A}} m_{1}\left(X_{1}\right) m_{2}\left(X_{2}\right)+ \\
\sum_{\substack{X \in 2^{\ominus} \\
X \cap=\emptyset}}\left[\frac{m_{1}(A)^{2} m_{2}(X)}{m_{1}(A)+m_{2}(X)}+\frac{m_{2}(A)^{2} m_{1}(X)}{m_{2}(A)+m_{1}(X)}\right]
\end{gathered}
$$

All fractions in (4) which have a denominator of zero are discarded. All propositions/sets in the formula are in canonical form. PCR5 is commutative and not associative but quasiassociative.

\section{Evidential Operations}

Evidence to support or refute design options in a decision making process can be extracted from numerous information sources including reports, journals and magazine articles. Some sources may be regarded more reliable or have a higher priority compared to others. It is important to manage these factors in the fusion process to reduce errors in reporting beliefs for decision options. Discounting methods can be applied to transform the beliefs of sources to reflect both the reliability and priority which are discussed below.

\section{A. Reliability Discounting Techniques}

Smarandache et al. [6] defined the reliability of an evidence source as its ability to provide the correct assessment/solution of the given problem. For example, a peer reviewed journal paper supporting a decision option could be regarded more reliable than a blog post. In reliability discounting a discounting factor $\alpha$ in $[0,1]$ can be applied to characterize the quality of an evidence source. This factor transforms the belief of each source to reflect it's credibility. The Shafer's discounting technique [2] has been proposed for the combination of unreliable evidence sources. Incorporation of the reliability factor $\alpha \in[0,1]$ in the decision making process is defined as:

$$
\left\{\begin{array}{r}
m_{\alpha}(X)=a \cdot m(X), \text { for } X \neq \theta \\
m_{\alpha}(\theta)=a \cdot m(\theta)+(1-\alpha)
\end{array}\right.
$$


whereby $\alpha=1$ represents a fully reliable source and $\alpha=0$ an unreliable source. The discounted mass is committed to the ignorance $m(\theta)$.

\section{B. Priority Discounting Technique}

The priority of an evidence source is distinct from the reliability of an evidence source. Applying the same definition of importance as [8] we regard source priority as an subjective attribute of an evidence source whereby a fusion designer/expert can assign a priority weight/value to individual source. For example, within the Aerospace domain safety is paramount therefore it is important to grant high priority to evidence sources containing information on aircraft safety. Within this paper we characterize priority using a priority factor denoted $\beta$ in $[0,1] . \beta$ is representative of a priority weight assigned by a fusion designer/expert to a source with a value between $[0,1]$. To consider priority in the fusion process we apply the priority discounting technique which is based upon the importance technique detailed in [6]. The idea of importance discounting was firstly presented by Tacnet in [7] and further explored by Smarandache et al. [6]. The approach defines discounting with respect to $\emptyset$ compared to $\Theta$ as used in the Shafer reliability approach detailed above. The highest priority assigned to a source is characterized by $\beta=1$ and the minimum priority $\beta=0$. The priority discounting of a source having a priority factor $\beta$ can be defined as:

$$
\left\{\begin{array}{r}
m_{\beta}(X)=\beta \cdot m(X), \text { for } X \neq \emptyset \\
m_{\beta}(\emptyset)=\beta \cdot m(\emptyset)+(1-\beta)
\end{array}\right.
$$

which allows $m(\emptyset) \geq 0$, thereby preserving specificity of the primary information as all focal elements are discounted with same priority factor [9].

The Dempster-Shafer Rule of Combination however cannot be used to fuse sources if priority discounting is applied. This is because the Dempster-Shafer Rule of Combination does not respond to the discounting of sources towards the empty set [6]. The PCR5 combination rule is therefore applied in this paper to demonstrate the fusion process when priority discounting is used. Using the priority technique the belief committed to $\emptyset$ is firstly normalized and then redistributed to the elements involved in the conflict proportionally to their individual masses.

\section{CAse Study}

In this section, we illustrate a decision making process whereby an engineer selects a material to construct a wing spar of an aircraft. This scenario is presented to help illustrate the underlying concepts and evidential operations of the proposed reasoning framework.

A spar is an integral structural member of the wing which extends through the length of the wing from the point closest to the fuselage to the wing tip. The function of the spar is to carry the flight loads and the weight of the wings [10]. Diverse materials including: wood, aluminum alloys and fiberreinforced composites can be used to construct a wing spar.
Using the proposed evidential framework we present how evidence varying in terms of reliability and priority that supports/opposes the design options: aluminum, composites and wood are fused together to aid an engineer in the decision making process. Prior knowledge has been applied to establish the weighting factors used by the reliability and priority discounting techniques.

\section{A. Standards, Requirements and Evidence}

In order to select a material to construct a wing spar, a number of factors need to be addressed. Firstly, the selected material must adhere to industry standards and secondly the material must fulfill specified design requirements. Information extracted from diverse sources is used to provide evidence that supports/refutes the various design options in fulfilling these criteria. An overview of the standards, requirements and evidence applied in this Case Study are presented below.

1) Standards: The selected material must be compliant with the Federal Aviation Administration (FAA) industry standard 25.651 (outlined in Table I) which requires proof of material strength before the aircraft can be certified for commercial use.

TABLE I

FAR 25.651 PROOF OF STRENGTH STANDARDS

\begin{tabular}{|l|}
\hline Part 25 AIRWORTHINESS STANDARDS: TRANSPORT CATE- \\
GORY AIRPLANES \\
\hline (a) Limit load tests of control surfaces are required. These tests must \\
include the horn or fitting to which the control system is attached. \\
\hline (b) Compliance with the special factors requirements of Secs. 25.619 \\
through 25.625 and 25.657 for control surface hinges must be shown \\
by analysis or individual load tests. \\
\hline
\end{tabular}

2) Requirements: The wing spar material must also fulfill design requirements such as safety, damage tolerance, cost and environmental impact. An aviation expert has assigned priory values to these requirements whereby essential requirements are assigned a higher priority compared to other less critical requirements. A weight factor of 1 represents a requirement of maximum priority and 0.2 of least priority.

3) Evidence: Evidence supporting and/or opposing the selection of materials have been extracted from heterogeneous sources. A total of 50 different sources were used which included: 18 journal articles, 6 technical white papers, 9 books, 7 aviation magazines and 10 blogs. These diverse sources varied in terms of certainty and consistency with the resulting knowledge base containing evidence some of which may be conflicting. Erroneous results could be produced if we assumed all evidence sources had the same reliability. To address this, reliability weights ranging from 0.3 to 0.9 were applied to the evidence sources based upon the credibility and certainty of the source. As the credibility of the source increases the reliability weight moves closer to 1 .

4) Evidence Vector: An evidence vector was constructed and used as input into the evidential reasoning framework. This vector was built by mapping the evidence for the design options to the relevant design requirements and standards 
which they either fulfilled or did not conform to. A sample of this evidence vector and the type of information that it contains is presented in Table II below.

TABLE II

SAMPLE EVIDENCE VECTOR

\begin{tabular}{|l|l|l|l|}
\hline & Aluminum & Composite & Wood \\
\hline Evidence & $\begin{array}{l}\text { Tolerant } \\
\text { material }\end{array}$ & $\begin{array}{l}\text { Damage } \\
\text { resistance }\end{array}$ & $\begin{array}{l}\text { Limited } \\
\text { availability }\end{array}$ \\
\hline Reliability & Journal (0.9) & Magazine (0.7) & Blog (0.3) \\
\hline Requirement & Safety & $\begin{array}{l}\text { Damage } \\
\text { Tolerance }\end{array}$ & Availability \\
\hline Priority & $\begin{array}{l}\text { High priority } \\
(1)\end{array}$ & High priority (1) & $\begin{array}{l}\text { Low priority } \\
(0.2)\end{array}$ \\
\hline
\end{tabular}

\section{B. Implementation of Scenario}

An engineer has the task of selecting one material from the set of materials: aluminum (A), composites (C) and wood (W) over the frame of discernment $\theta=\{\emptyset, A, C, W\}$, i.e. Shafer's model, from which to construct a wing spar. For simplistic purposes, we assume that the selected material only needs to fulfill the requirements: safety, damage tolerance and availability of supply as detailed in Table II. We use four different evidence sources that assign belief to the hypotheses. The estimated respective bba's: $m_{1}, m_{2}, m_{3}$ and $m_{4}$ are described in Table III. The mass affectation is estimated using information from the constructed knowledge base along with expert knowledge.

TABLE III

Basic Belief Assignments For Evidence Sources

\begin{tabular}{|l|l|l|l|l|}
\hline & $m_{1}$ & $m_{2}$ & $m_{3}$ & $m_{4}$ \\
\hline$A$ & 0.4 & 0.2 & 0.8 & 0.5 \\
$C$ & 0.4 & 0.7 & 0.1 & 0.4 \\
$W$ & 0.1 & 0.01 & 0.01 & 0.05 \\
$A \cup C \cup W$ & 0.1 & 0.09 & 0.09 & 0.05 \\
\hline
\end{tabular}

Reliability weightings for the evidence have been derived using source information from which the evidence was extracted. For example, evidence obtained from a peer reviewed journal has a high reliability factor compared to a blog post where information provided may not be scientifically verified. The reliability factors for the four sources are as follows: $\alpha_{1}=0.9, \alpha_{2}=0.7, \alpha_{3}=0.3, \alpha_{4}=0.3$. The Aerospace engineer who has specified the requirements has also ranked them in order of priority whereby a requirement considered high priority is set with a higher discounting factor. The priority factors for the respective four sources are: $\beta_{1}=1, \beta_{2}=1, \beta_{3}=0.6, \beta_{4}=0.2$.

To fuse the heterogeneous sources we use the PCR5 and Dempster-Shafer rules of combination. Based on the simplified scenario, both the reliability and the priority discounting techniques are applied and analyzed individually. When applying the priority discounting technique only results obtained using the PCR5 rule of combination are presented as the DempsterShafer rule of combination does not respond to the discounting of sources towards the empty set. [6].
1) PCR5 and Dempster-Shafer Rules of Combination: We firstly present the scenario whereby evidence was fused using the PCR5 and Dempster-Shafer rules of combination based on the assumption that all sources are equal in terms of reliability and priority. The results obtained for this scenario are shown in Table IV and Table V. In the first row of the Tables IV and $\mathrm{V} m_{12} \ldots, m_{1234}$ corresponds to the sequential fusion of the sources $m_{1} \ldots, m_{4}$. We can see from Table III that $m_{2}$ and $m_{3}$ are conflicting sources as the bba $m_{2}$ assigns most belief to hypothesis $C$ compared to $m_{3}$ where most of the belief is assigned to $A$. Based on the results highlighted in Tables IV and V both PCR5 and Dempster-Shafer rules of combination assigned the highest belief to the hypothesis $A$. Interestingly, there is a noticeable different between the distribution of beliefs across the hypotheses $A$ and $C$ by these rules. The Dempster-Shafer rule of combination assigns more belief to $A$ and less to $C$ in comparison to the PCR5 rule of combination. The difference between these results can be explained by the approaches used by the combination rules to distribute conflict. The Dempster-Shafer rule distributes equally the total conflicting mass over all focal elements of $2^{\Theta}$ (including elements that may not have contributed to the conflict) whereas PCR5 transfers conflicting masses proportionally to non-empty sets involved in the model. This highlights the importance high conflict can have on belief assignment when DS is applied. [11].

TABLE IV

DEMPSTER-SHAFER RULE OF COMBINATION RESUlTS WHEN ALL SOURCES ARE AsSUMEd EQUAL

\begin{tabular}{|l|c|c|c|}
\hline & $m_{12}$ & $m_{123}$ & $m_{1234}$ \\
\hline$A$ & 0.2509 & 0.6272 & $\mathbf{0 . 6 7 6 4}$ \\
$C$ & 0.7122 & 0.3631 & 0.3217 \\
$W$ & 0.0203 & 0.0058 & 0.0015 \\
$A \cup C \cup W$ & 0.0166 & 0.0040 & $3.8620 \mathrm{E}-4$ \\
\hline
\end{tabular}

TABLE V

PCR5 Rule of COMbination Results When All Sources aRe ASSUMED EQUAL

\begin{tabular}{|l|c|c|c|}
\hline & $m_{12}$ & $m_{123}$ & $m_{1234}$ \\
\hline$A$ & 0.2817 & 0.5967 & $\mathbf{0 . 6 1 2 0}$ \\
$C$ & 0.6827 & 0.3983 & 0.3830 \\
$W$ & 0.0267 & 0.0042 & 0.0050 \\
$A \cup C \cup W$ & 0.0090 & $8.1 \mathrm{E}-4$ & $4.05 \mathrm{E}-5$ \\
\hline
\end{tabular}

2) Reliability Discounting: In Tables VI and VII we present results obtained when the reliability discounting technique is implemented with the combination rules approaches Dempster-Shafer and PCR5 respectively. The application of reliability discounting to the bba's reduces in particular the beliefs in $m_{3}$ and $m_{4}$. Compared to the results in Tables IV and $\mathrm{V}$, both combination rules now assign most belief to the hypothesis $C$ when reliability factors are considered. It is known that conflicts can have a counter-intuitive impact on the results obtained using the Dempster-Shafer rule of combination if conflict between hypotheses are high [12]. However, by applying reliability discounting factors the degree 
of conflict between the bba's: $m_{2}$ and $m_{3}$ was reduced by committing the discounted mass to ignorance. This could explain why similar belief assignments are now observed across the two fusion approaches.

TABLE VI

DEMPSTER-SHAFER RULE OF COMBINATION RESULTS WHEN RELIABILITY DisCOUNTING IS APPLIED

\begin{tabular}{|l|c|c|c|}
\hline & $m_{12}$ & $m_{123}$ & $m_{1234}$ \\
\hline$A$ & 0.3460 & 0.3845 & 0.4030 \\
$C$ & 0.5084 & 0.4921 & 0.4917 \\
$W$ & 0.0487 & 0.0416 & 0.0369 \\
$A \cup C \cup W$ & 0.0969 & 0.0818 & 0.0683 \\
\hline
\end{tabular}

TABLE VII

PCR5 RULE OF COMBINATION RESULTS WHEN RELIABILITY DISCOUNTING IS APPLIED

\begin{tabular}{|l|c|c|c|}
\hline & $m_{12}$ & $m_{123}$ & $m_{1234}$ \\
\hline$A$ & 0.3595 & 0.3963 & 0.4145 \\
$C$ & 0.5244 & 0.5158 & 0.5187 \\
$W$ & 0.0471 & 0.0378 & 0.0310 \\
$A \cup C \cup W$ & 0.0690 & 0.0501 & 0.0359 \\
\hline
\end{tabular}

3) Priority Discounting: We have demonstrated the importance of reliability factors and their impact on the decision making process. We now present results obtained using the priority discounting technique. This technique differs to the reliability discounting technique as the discounted mass is assigned to the empty set compared to $\Theta$. The impact of this approach is demonstrated using the PCR5 rule of combination. Both $m_{1}$ and $m_{2}$ were identified as the highest priority bba's. By applying priority discounting to $m_{3}$ and $m_{4}$ we can view the impact on the decision making process in Table VIII.

TABLE VIII

PCR5 RULE OF COMBINATION WITH PRIORITY DisCOUNTING

\begin{tabular}{|l|c|c|c|}
\hline & $m_{12}$ & $m_{123}$ & $m_{1234}$ \\
\hline$A$ & 0.2817 & 0.4965 & 0.4765 \\
$C$ & 0.6827 & 0.4988 & 0.5163 \\
$W$ & 0.02662 & 0.0042 & 0.0072 \\
$A \cup C \cup W$ & 0.0090 & $4.8510 \mathrm{E}-4$ & $1.45710 \mathrm{E}-5$ \\
\hline
\end{tabular}

Compared to the fusion results in Table $\mathrm{V}$ where no discounting was performed, a significant difference in belief assignment can be seen from Table VIII when priority factors are applied. Furthermore, using the same weighting factors, a notable difference is observed between the priority discounting results in Table VIII and reliability discounting in Table IX.

TABLE IX

PCR5 RULE OF COMBINATION WITH RELIABILITY DISCOUNTING

\begin{tabular}{|l|c|c|c|}
\hline & $m_{12}$ & $m_{123}$ & $m_{1234}$ \\
\hline$A$ & 0.2817 & 0.4304 & 0.4027 \\
$C$ & 0.6827 & 0.5520 & 0.5837 \\
$W$ & 0.0266 & 0.0135 & 0.0111 \\
$A \cup C \cup W$ & 0.0090 & 0.0041 & 0.0026 \\
\hline
\end{tabular}

These scenario results have demonstrated how reliability and priority discounting techniques can provide interesting results and may be beneficial when integrated within decision making support systems. The application choice of the two discounting rules is left to the users accordingly to their own purposes.

\section{CONClusion}

Decisions made throughout the life-cycle of large-scale projects are essential as they have a direct impact upon the outcome and success of a project. Aerospace is an example problem space that spans many domains whereby decisions are often made in the presence of information which may be noisy, conflicting, incomplete and which varies in terms of reliability and priority. To address these issues, we presented an evidential reasoning framework to aid in the decision making process illustrated by a Case Study based in the Aerospace domain. This framework incorporated knowledge on the reliability and priority of evidential sources to show their impact and importance upon the decision making process. Furthermore, we illustrated how unreliable and diverse priorities can be accommodated in the decision making process. To evaluate this approach we focused on applying DSm to fuse uncertain evidential sources and to also incorporate the reliability and our proposed priority discounting technique. For comparative purposes only we presented DS, this was due to the potential of DS generating errors in the decision making process when paradoxical information and conflict between hypotheses becomes high. We also described how the priority discounting technique cannot be applied using the Dempster-Shafer rule of combination contrariwise to the PCR5 rule used in by the DSm approach. Through the examples outlined in the Aerospace Case Study presented in this paper, we illustrated the potential interest of the evidential framework along with discounting techniques for decision-making in practical scenarios.

\section{ACKNOWLEDGMENTS}

The DEEPFLOW project is funded by Invest Northern Ireland ref: RD1208002 and SAP (AG).

\section{REFERENCES}

[1] W. Yao, X. Chen, W. Luo, M. van Tooren, and J. Guo, "Review of uncertainty-based multidisciplinary design optimization methods for aerospace vehicles," Progress in Aerospace Sciences, vol. 47, no. 6, pp. $450-479,2011$

[2] G. Shafer, A mathematical theory of evidence. Princeton university press Princeton, NJ, 1976, vol. 1.

[3] H. Lee, J. S. Choi, and R. Elmasri, "Sensor data fusion using dsm theory for activity recognition under uncertainty in home-based care," in Advanced Information Networking and Applications, 2009. AINA '09. International Conference on, may 2009, pp. 517 -524.

[4] J. Dezert, F. Smarandache, and M. Daniel, "The generalized pignistic transformation," Arxiv preprint cs/0409007, 2004.

[5] S. Corgne, L. Hubert-Moy, J. Dezert, and G. Mercier, "Land cover change prediction with a new theory of plausible and paradoxical reasoning," in Proc. of Fusion, 2003, pp. 8-11.

[6] F. Smarandache, J. Dezert, and J. Tacnet, "Fusion of sources of evidence with different importances and reliabilities," in Information Fusion (FUSION), 2010 13th Conference on. IEEE, 2010, pp. 1-8.

[7] J. Smarandache, F. Dezert, Ed., Advances and Applications of DSmT for Information Fusion. American Research Press, Rehoboth, 2009, vol. Vol.1-3, 
[8] J. Dezert, J. Tacnet, M. Batton-Hubert, and F. Smarandache, "Multicriteria decision making based on dsmt-ahp," 2010.

[9] Z. Liu, J. Dezert, Q. Pan, and G. Mercier, "Combination of sources of evidence with different discounting factors based on a new dissimilarity measure," Decision Support Systems, vol. 52, no. 1, pp. 133 - 141, 2011. [Online]. Available: http://www.sciencedirect.com/science/article/pii/S0167923611001187

[10] J. Rao, S. Kiran, S. Chandra, J. Kamesh, and M. Padmanabhan, "Topology optimization of aircraft wing," in Proceedings of the Altair India/S. Asia HyperWorks Technology Conference, 2008.

[11] J. Dezert, A. Tchamova, F. Smarandache, and P. Konstantinova, "Target type tracking with pcr5 and dempster's rules: A comparative analysis," Arxiv preprint cs/0607143, 2006.

[12] A. Josang, "The consensus operator for combining beliefs," Artificial Intelligence, vol. 141 , no. 1-2, pp. 157-170, 2002. 